\title{
THE ENERGY SAVING STRATEGY ON THE SUSTAINABLE CAMPUS RENOVATION PLAN BY RECYCLING AND REUSE OF RAINWATER IN TAIWAN
}

\author{
LIAO, Y. T.- LIU, K. S. \\ Department of Interior Design, Tung Fang Design Institute \\ No.110, Dongfang Rd., Hunei Dist., Kaohsiung City 82941, Taiwan (R.O.C.) \\ *Corresponding author \\ e-mail: kliu1219@yahoo.com.tw; tel: +886-9-287-51652; fax: +886-7-693-9657 \\ (Received $12^{\text {th }}$ Aug 2016; accepted $15^{\text {th }}$ Nov 2016)
}

\begin{abstract}
The main purpose of this study is to investigate the energy saving strategy on the Sustainable Campus Renovation Plan by recycling and reuse of rainwater in Taiwan. Case studies from the past were also investigated for effectiveness comparisons, which include both economical values and environmental values. Data Envelopment Analysis (DEA) has been implemented for quantification purposes and it provided the visualization and comparison of energy saving improvement database. Moreover, a model for assessing the improvement on the recycling and reuse of rain water could be constructed. In addition, a set of improved strategy process and steps of rain water recycling and reuse methodology may be proposed which result in the vision of the Sustainable Campus Renovation establishment.
\end{abstract}

Keywords: sustainable school, rainwater recycle, rainwater reuse, energy, Data Envelopment Analysis (DEA)

\section{Introduction}

In Chapter 36 of Agenda 21, compiled in Rio 1992, a reference is made to: "Education, raising of public awareness and training are linked to virtually all areas in Agenda 21, and even more closely to the ones on meeting basic needs, capacitybuilding, data and information, science and the role of major groups. Education, including formal education and training, should be recognized as a process by which human beings and societies can reach their fullest potential".

The Sustainable Campus Renovation Plan by the Ministry of Education, Taiwan has been carried out for 13 years based on two major topics which include the "environmental suitability" and the "sustainable education". School teachers and students proactively identified campus environments which are unfriendly, ineffective in energy saving, and not sustainable. Therefore, proposals on the improved countermeasures have occurred via brainstorming. In addition, these have been implemented to the participant-centered learning program during the beginning, mid-term, and final stage of reengineering process and have integrated the improved result into the academic circle to enable school students to personally feel the wonderfulness of environmental re-establishment and truly identify the importance of sustainability. Thus, schoolyards needs an appropriate assessment tool to examine the intrinsic and extrinsic effects with regard to the Environmental Protection and Ecology (Liao et al., 2014).

It is known from the average annual rainfall that the water resource is unevenly distributed in Taiwan. Therefore, drought occurs easily and the Ministry of Education has proactively promoted the concept of correctly using the water resources, and request school to assess water facilities progressively and to improve budget planning on a 
yearly basis. Furthermore, in order to save valuable water resources, the Sustainable Campus Renovation Plan by the Ministry of Education provides subsidies to improve the facilities on rainwater recycle system, turning the output of the system into restroom flush or plants watering. Growing water scarcity and global climate change both call for more efficient alternatives of water conservation. The rainwater harvesting (RWH) is the most promising alternative among others. (Magliano et al., 2015). This study fully utilizes the result from a past case study of campus sustainability as the database and consolidates the energy-saving technology (rainwater recycling and reuse) which the Sustainable Campus Renovation Plan proposed by the Ministry of Education, Taiwan in an attempt to promote the investigation of the pros and cons of the energy-saving efficiency. Therefore, the results lead to the construction of energy-saving efficiency assessment model and the establishment of improved energy-saving strategies. This assessment model and strategy shall therefore be considered as one of the most significant factors in the future renovation of the school.

\section{Materials and methods}

This study has collected data from campuses which have rainwater recycle improvement case and impose the view of environmental efficiency. The key factors include improving the energy saving (input) / assessment factor as the output. With this opportunity, it is expected to better understand the technique of localized energy-saving improvement. In order to provide relevant scientific facts and a quantitative analysis and to provide the most suitable energy-saving strategy for school building in Taiwan, this study used the Data Envelopment Analysis (DEA) of Multiple-Criteria Decision Analysis (MCDA) as the main analytical method. In addition, the result shall prevent campus management and designer from selecting poor energy-saving techniques and to avoid the waste of resources and money.

\section{Selection of analytical modes}

Recently, some studies have applied DEA to macro performance assessment, energy control solutions and cost-benefit empirical models in order to improve overall efficiencies (Macek and Marik, 2012; Chang and Yang, 2011). DEA has recently become a popular approach for measuring the energy and environmental performance at the macro-economy level (Wang et al., 2013; Yu and Chan, 2012; Blomberg et al., 2012).

Traditional CCR model and BCC model are the measure of radial efficiency. These two models hypothesize that input and output could be increased or decreased proportionally. During the calculation process, a decision has to be made whether it is input or output orientated, and could not take both into consideration at the same time. Tone (2001) proposed the SBM (Slack-Based Measure) model in which variance variables are taken into consideration as the measurement fundamental. The assessment model of SBM is based on non-radial, and it takes into consideration the variance for both the input and the output. This amends not only the shortage of CCR model and BCC model (measure of radial efficiency) but also the drawback of unit invariance. Song et al. (2013) raised the new model's computing conclusions are highly related to the efficiency assessment of the DEA-SBM model. Moreover, his study emphasized more on the affects of undesirable outputs on production efficiency than the latter. This 
means the new model has a greater extensive value for application and provides a better quantitative theoretical basis for environmental policy analysis.

In accordance with the derivation by Tone (2001), the efficiency value derived from SBM model will not exceed the efficiency value derived from the CCR model. As for units which have been assessed, the SBM efficiency value $(\rho=1)$ is equivalent to the CCR model's efficiency value. Therefore, the SBM model has been selected as the analytical model for the efficiency measurement. The fractional linear programming of the SBM efficiency value is illustrated by Eq. 1 as follows:

$$
\begin{gathered}
\min \rho=\frac{1-\frac{1}{m} \sum_{i=1}^{m} s_{i}^{-} / x_{i o}}{1+\frac{1}{s} \sum_{r=1}^{s} s_{r}^{+} / y_{r o}} \\
\text { s.t. } \quad x_{o}=X \lambda+s^{-} \\
y_{o}=Y \lambda-s^{+} \\
\lambda, s^{-}, s^{+} \geq 0
\end{gathered}
$$

In Eq. $1, \rho$ is variance index of non-radial, si- and si+ represent variance and output variance, $X \lambda \& Y \lambda$ represent the benchmark of efficient frontier for both input and output. If we assume $X \geq 0$ and if $x i o=0$ then si-/xio has to be removed from the objective function. In order for si+/yro to highlight the negative impact on the SBM efficiency, yro has to be replaced by minimum positive value when yro $\leq 0.0 \leq \rho \leq 1$ represent SBM efficiency value is being limited between $0 \sim 1$. Since $\rho$ uses the index constructed by both si-and si+, when all of the values of si- and si+ are 0 it indicates that all the input and output values of DMU present no variance. Therefore, when $\rho=1$ we can conclude that DMU has SBM efficiency.

\section{The interpretation of the result}

Since DEA models are vulnerable to the attributes of inputs and outputs and the data of DMUs, this study employs both the efficiency and the susceptibility analyses to identify the changes and causes of respective decision-making units besides the SBM for improved validity.

Efficiency analysis: According to Chang et al. (1995) the efficiency refers to the level with which an objective is achieved. We compute the efficiency scores of respective DMUs by substituting 1 as the input of DEA.

Susceptibility analysis: We identified how the changes of inputs and outputs affect the relative efficiency scores of respective DMUs and ascertain the affected levels of respective outputs on a DMU by excluding the SBM result of a specific output and observing how the efficiency scores change accordingly. Table 1 illustrates the method of sensitivity analysis. 
Table 1. Data analysis

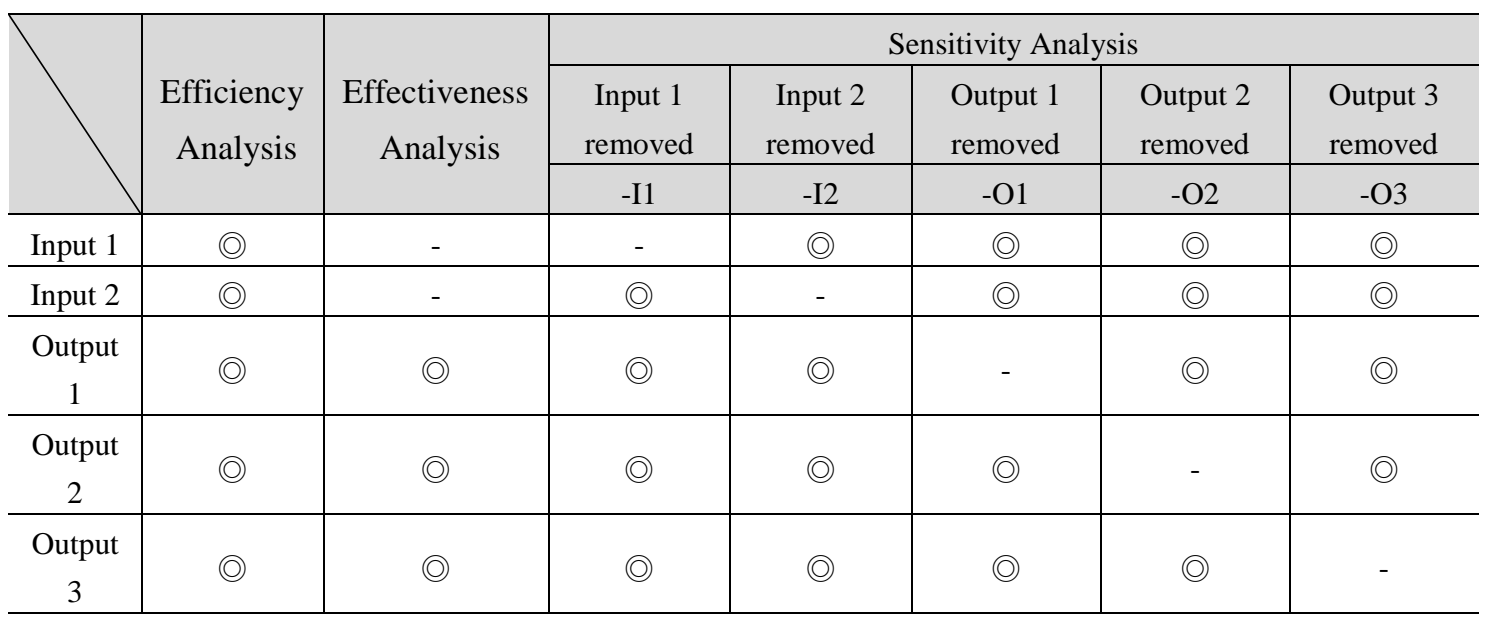

Remark: () Impose SBM model into the calculation of input and output.

\section{Results and discussion}

\section{Investigations on the energy-saving efficiency of rainwater recycling and reuse system by using DEA}

Rainwater recycling and reuse subsidy program under the Sustainable Campus Renovation Plan by the Ministry of Education, Taiwan has experienced several stages of turning points. During the early stage of the subsidy, schools have proactively recycled and centralized water source collection in an attempt to cope with improvements on rainwater reusability plan. However, these water sources consist of rainwater, water for washing hands, water for cleaning vegetables, RO water, spring water etc. since water came from different sources with different pollution/contamination levels. Therefore, using a simple filtration system does not guarantee an effective management of those recycled water. In addition, this could result in water pollution/contamination and could lead to issues which may cause school extra efforts in maintenance management. Rainwater is only one of the sources of recycle water. There are other water sources and these are mainly from used tap water or groundwater. If these were to be recycled, pollution/contamination risk shall be taken into consideration

In view of this, from its early stage. the reformation of recycling water under sustainable campus program needs to have dynamic transformation techniques on ecological pool, kitchen water and grease separation, rainwater recycling and reuse. However, due to the consideration of "water quality management", the program has recently been constrained to the recycling and reuse of rainwater only. Although the source of rainwater is not as complex as other sources, the transformation technique which is derived from rainwater treatment is not anything less. In the early phrase, a single water tank was used for water collection purpose, then changed to "gravity water supply". The purpose of this approach is to have a recycling water tower located on top floor, and to better utilize the school buildings' raft foundation for water collection purpose. Moreover, underground excavations in school area were carried out to set up water collection tank. Therefore, high ground water tank, underground RC water tank, and raft foundation water tank are the major three significant factors which this study investigate upon on the efficiency of rainwater recycling and reuse. The water quality of 
an ecological pool is difficult to maintain and manage. Moreover, due to safety issues and the instability of water sources during different seasons, the campus sustainable program currently does not encourage this to be included in the program and thus they have been excluded throughout this study.

With reference to this topic, the purpose of this study is to investigate and compare the efficiency of recycling and reuse on "High ground water tank (recycling \& reuse)", "underground RC water tank collection", "high ground water tank with rafted foundation". Based on the above clarification, this study will set "early phrase establishment cost (dollar)", and "estimated maintenance management cost (dollar/year)" as the input, and "area of rainwater collection $\left(\mathrm{m}^{2}\right)$ ", "water storage capacity (ton)", and "re-utilization (ton)"as the output (Table 2). SBM-O-C model has been selected to calculate the improved efficiency value. The definition of output will be used in accordance to Clause 2, Article 309 under the Construction Design of Building Technical Regulations. The collection and calculation conversion of input and output are clarified as follows:

(I1) Early phrase establishment cost (dollar): actual cost of engineering contracts for that particular year. It is calculated in dollars.

(I2) Estimated maintenance management cost (dollar/year): Schools were asked to estimate the budget required for day-to-day maintenance and management. It is calculated in dollars.

(O1) Area of rain water collection $\left(\mathrm{m}^{2}\right)$ : The area of rain water collection from the use of facilities and buildings, for instead, the projected roof or canopy.

(O2) Water storage capacity (ton): The amount of rain water stored from the use of water storage tank and building. This excluded the facility which runs water quality treatment process.

(O3) Re-utilization (ton): The amount of rain water stored from the use of facilities and effective rainwater utilization. This is calculated in tons and rounded up to one digit after the decimal point. In order to cope with the data of the Sustainable Campus Renovation Plan by the Ministry of Education provided by each school, the reutilization statistical data will be based on May-Aug of the coming year upon on the completion of the improvement.

Table 2. Rainwater (Recycling \& Reuse)- Original Data

\begin{tabular}{|c|c|c|c|c|c|c|c|c|c|}
\hline \multirow[b]{3}{*}{$\begin{array}{l}\text { DMU } \\
\text { Code }\end{array}$} & \multirow[b]{3}{*}{ Recycling Method } & \multirow[b]{3}{*}{$\begin{array}{l}\text { Motor } \\
\text { Usage }\end{array}$} & \multirow[b]{3}{*}{$\begin{array}{l}\text { Filtrations } \\
\text { Facility }\end{array}$} & \multirow[b]{3}{*}{$\begin{array}{l}\text { Re-utilization } \\
\text { method }\end{array}$} & \multicolumn{5}{|c|}{ Original Data } \\
\hline & & & & & (I1) & (I2) & $(\mathrm{O} 1)$ & $(\mathrm{O} 2)$ & $(\mathrm{O} 3)$ \\
\hline & & & & & 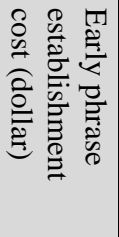 & 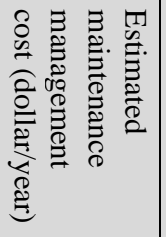 & 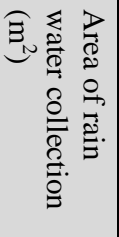 & 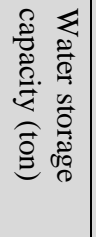 & 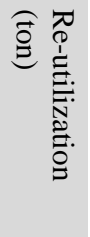 \\
\hline 1 & PVC bucket & NO & Filter & Watering & 27560 & 150 & 50 & 1.12 & 61 \\
\hline 2 & $\begin{array}{l}\text { Stainless Water } \\
\text { Tower }\end{array}$ & YES & $\begin{array}{l}\text { Rainwater } \\
\text { Sedimentat } \\
\text { ion Tank }\end{array}$ & $\begin{array}{l}\text { Flushing toilet, } \\
\text { Watering, } \\
\text { floor cleaning }\end{array}$ & 723600 & 1500 & 900 & 22 & 1720 \\
\hline 3 & $\begin{array}{l}\text { Stainless Water } \\
\text { Tower }\end{array}$ & NO & Filter & $\begin{array}{l}\text { Watering, } \\
\text { rehydrate }\end{array}$ & 52000 & 200 & 209 & 3 & 90 \\
\hline
\end{tabular}




\begin{tabular}{|c|c|c|c|c|c|c|c|c|c|}
\hline \multirow[b]{3}{*}{$\begin{array}{l}\text { DMU } \\
\text { Code }\end{array}$} & \multirow[b]{3}{*}{ Recycling Method } & \multirow[b]{3}{*}{$\begin{array}{l}\text { Motor } \\
\text { Usage }\end{array}$} & \multirow[b]{3}{*}{$\begin{array}{c}\text { Filtrations } \\
\text { Facility }\end{array}$} & \multirow[b]{3}{*}{$\begin{array}{l}\text { Re-utilization } \\
\text { method }\end{array}$} & \multicolumn{5}{|c|}{ Original Data } \\
\hline & & & & & (I1) & (I2) & $(\mathrm{O} 1)$ & $(\mathrm{O} 2)$ & $(\mathrm{O} 3)$ \\
\hline & & & & & 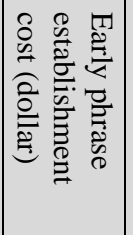 & 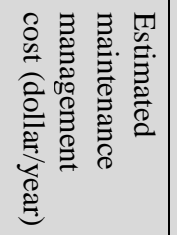 & 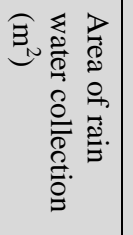 & 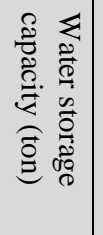 & 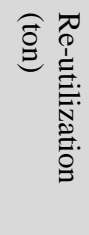 \\
\hline & & & & wetland & & & & & \\
\hline 4 & $\begin{array}{l}\text { Stainless Water } \\
\text { Tower }\end{array}$ & NO & $\begin{array}{l}\text { Filter } \\
\text { Basic Filter } \\
\text { Tank }\end{array}$ & Watering & 115000 & 300 & 946 & 10 & 300 \\
\hline 5 & $\begin{array}{l}\text { Stainless Water } \\
\text { Tower }\end{array}$ & NO & NO & $\begin{array}{l}\text { Watering, } \\
\text { rehydrate } \\
\text { wetland }\end{array}$ & 98000 & 300 & 720 & 10 & 245 \\
\hline 6 & $\begin{array}{l}\text { Stainless Water } \\
\text { Tower }\end{array}$ & NO & $\mathrm{NO}$ & Watering & 564000 & 1500 & 652 & 15 & 604 \\
\hline 7 & $\begin{array}{l}\text { Stainless Water } \\
\text { Tower }\end{array}$ & YES & NO & $\begin{array}{l}\text { Watering, } \\
\text { rehydrate } \\
\text { wetland }\end{array}$ & 60000 & 200 & 200 & 5 & 160 \\
\hline 8 & $\begin{array}{l}\text { Stainless Water } \\
\text { Tower }\end{array}$ & YES & Filter & $\begin{array}{l}\text { Watering, } \\
\text { rehydrate } \\
\text { wetland }\end{array}$ & 380000 & 1000 & 306 & 6 & 312 \\
\hline 9 & $\begin{array}{l}\text { Stainless Water } \\
\text { Tower }\end{array}$ & NO & NO & Watering & 278500 & 750 & 1170 & 15 & 350 \\
\hline 10 & $\begin{array}{l}\text { Stainless Water } \\
\text { Tower }\end{array}$ & NO & $\begin{array}{l}\text { Basic Filter } \\
\text { Tank } \\
\text { Filter }\end{array}$ & Watering & 319200 & 800 & 1265 & 20 & 210 \\
\hline 11 & $\begin{array}{l}\text { Stainless Water } \\
\text { Tower }\end{array}$ & NO & NO & Watering & 665000 & 200 & 640 & 10 & 65 \\
\hline 12 & $\begin{array}{l}\text { Stainless Water } \\
\text { Tower }\end{array}$ & YES & NO & Watering & 435886 & 1200 & 567 & 15 & 360 \\
\hline 13 & $\begin{array}{l}\text { Stainless Water } \\
\text { Tower }\end{array}$ & YES & $\begin{array}{l}\text { Sedimentat } \\
\text { ion pool }\end{array}$ & Watering & 205000 & 500 & 782 & 10 & 165 \\
\hline 14 & $\begin{array}{l}\text { Stainless Water } \\
\text { Tower }\end{array}$ & YES & $\mathrm{NO}$ & Watering & 835045 & 200 & 60 & 3 & 56 \\
\hline 15 & $\begin{array}{l}\text { Stainless Water } \\
\text { Tower }\end{array}$ & NO & $\mathrm{NO}$ & Watering & 66000 & 200 & 360 & 10 & 43 \\
\hline 16 & $\begin{array}{l}\text { Stainless Water } \\
\text { Tower }\end{array}$ & NO & NO & $\begin{array}{l}\text { Flushing toilet, } \\
\text { Watering }\end{array}$ & 260000 & 600 & 2100 & 15 & 310 \\
\hline 17 & $\begin{array}{l}\text { Stainless Water } \\
\text { Tower }\end{array}$ & NO & NO & Watering & 40000 & 100 & 160 & 5 & 42 \\
\hline
\end{tabular}




\begin{tabular}{|c|c|c|c|c|c|c|c|c|c|}
\hline \multirow[b]{3}{*}{$\begin{array}{l}\text { DMU } \\
\text { Code }\end{array}$} & \multirow[b]{3}{*}{ Recycling Method } & \multirow[b]{3}{*}{$\begin{array}{l}\text { Motor } \\
\text { Usage }\end{array}$} & \multirow[b]{3}{*}{$\begin{array}{c}\text { Filtrations } \\
\text { Facility }\end{array}$} & \multirow[b]{3}{*}{$\begin{array}{l}\text { Re-utilization } \\
\text { method }\end{array}$} & \multicolumn{5}{|c|}{ Original Data } \\
\hline & & & & & (I1) & (I2) & $(\mathrm{O} 1)$ & $(\mathrm{O} 2)$ & $(\mathrm{O} 3)$ \\
\hline & & & & & 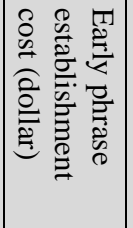 & 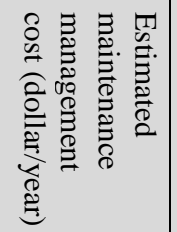 & 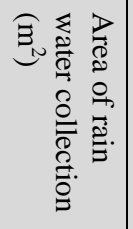 & 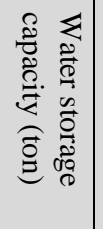 & 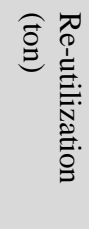 \\
\hline 18 & $\begin{array}{l}\text { underground RC } \\
\text { water tank } \\
\text { collection }\end{array}$ & YES & $\begin{array}{l}10 \mathrm{M} \\
\text { Ecological } \\
\text { river }\end{array}$ & $\begin{array}{l}\text { Watering, } \\
\text { rehydrate } \\
\text { wetland }\end{array}$ & 580000 & 1500 & 507 & 68 & 528 \\
\hline 19 & $\begin{array}{l}\text { underground RC } \\
\text { water tank } \\
\text { collection+ } \\
\text { water tower }\end{array}$ & YES & $\begin{array}{l}\text { Sedimentat } \\
\text { ion pool }\end{array}$ & Watering & 216000 & 600 & 882 & 10 & 220 \\
\hline 20 & $\begin{array}{l}\text { underground RC } \\
\text { water tank } \\
\text { collection } \\
\end{array}$ & YES & NO & Watering & $\mid 281760$ & 700 & 781 & 15 & 322 \\
\hline 21 & $\begin{array}{l}\text { underground RC } \\
\text { water tank } \\
\text { collection+ } \\
\text { water tower } \\
\end{array}$ & YES & $\begin{array}{l}\text { Filtration } \\
\text { tank }\end{array}$ & $\begin{array}{l}\text { Flushing toilet, } \\
\text { Watering }\end{array}$ & 186074 & 500 & 500 & 31 & 204 \\
\hline 22 & $\begin{array}{l}\text { Rafted } \\
\text { foundation + } \\
\text { water tower }\end{array}$ & YES & $\begin{array}{l}\text { Sedimentat } \\
\text { ion pool }\end{array}$ & Watering & |230000 & 600 & 542 & 300 & 460 \\
\hline 23 & $\begin{array}{l}\text { Rafted } \\
\text { foundation }\end{array}$ & YES & Filter & $\begin{array}{l}\text { Flushing toilet, } \\
\text { Watering, } \\
\text { fire hydrant }\end{array}$ & $\mid 160000$ & 500 & 1206 & 306 & 1118 \\
\hline
\end{tabular}

Table 3 refers more detail in the rainwater recycle improvement efficiency rate for "High ground water tank (recycling and reuse)", "underground RC water tank collection", "high ground water tank with rafted foundation". 
Table 3. Rainwater (Recycling \& Reuse)- Efficiency Analysis, Effectiveness Analysis and Sensitivity Analysis

\begin{tabular}{|c|c|c|c|c|c|c|c|c|c|}
\hline \multirow[b]{3}{*}{$\begin{array}{l}\text { DMU } \\
\text { Code }\end{array}$} & \multirow[b]{3}{*}{$\begin{array}{l}\text { Recycling } \\
\text { Method }\end{array}$} & \multirow[b]{3}{*}{ Case Group } & \multirow[b]{3}{*}{$\begin{array}{r}\text { Efficiency } \\
\text { Analysis }\end{array}$} & \multirow[b]{3}{*}{$\begin{array}{c}\text { Effectiveness } \\
\text { Analysis }\end{array}$} & \multicolumn{5}{|c|}{ Sensitivity Analysis } \\
\hline & & & & & $-\mathrm{I} 1$ & $-\mathrm{I} 2$ & $-\mathrm{O} 1$ & $-\mathrm{O} 2$ & $-\mathrm{O} 3$ \\
\hline & & & & & 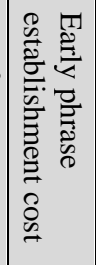 & 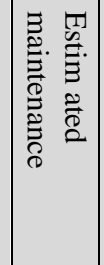 & 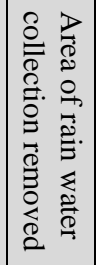 & 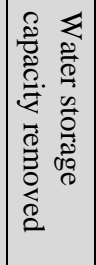 & 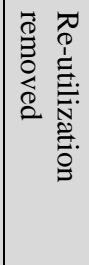 \\
\hline & PVC bucket & \multirow{16}{*}{$\begin{array}{c}\text { High Ground } \\
\text { water tower } \\
\text { (Recycling \& } \\
\text { Reuse) }\end{array}$} & 0.055 & 0.010 & 0.032 & 0.055 & 0.040 & 0.274 & 0.039 \\
\hline 2 & $\begin{array}{l}\text { Stainless Water } \\
\text { Tower }\end{array}$ & & 0.063 & 1.000 & 0.063 & 0.042 & 0.046 & 0.324 & 0.044 \\
\hline 3 & $\begin{array}{l}\text { Stainless Water } \\
\text { Tower }\end{array}$ & & 0.077 & 0.025 & 0.062 & 0.077 & 0.054 & 0.338 & 0.057 \\
\hline 4 & $\begin{array}{l}\text { Stainless Water } \\
\text { Tower }\end{array}$ & & 1.000 & 0.084 & 0.356 & 1.000 & 0.097 & 1.000 & 1.000 \\
\hline 5 & $\begin{array}{l}\text { Stainless Water } \\
\text { Tower }\end{array}$ & & 0.136 & 0.081 & 0.136 & 0.133 & 0.095 & 0.534 & 0.103 \\
\hline 6 & $\begin{array}{l}\text { Stainless Water } \\
\text { Tower }\end{array}$ & & 0.041 & 0.124 & 0.041 & 0.035 & 0.030 & 0.180 & 0.030 \\
\hline 7 & $\begin{array}{l}\text { Stainless Water } \\
\text { Tower }\end{array}$ & & 0.108 & 0.040 & 0.101 & 0.108 & 0.078 & 0.410 & 0.079 \\
\hline 8 & $\begin{array}{l}\text { Stainless Water } \\
\text { Tower }\end{array}$ & & 0.026 & 0.051 & 0.026 & 5.022 & 0.018 & 0.133 & 0.018 \\
\hline 9 & $\begin{array}{l}\text { Stainless Water } \\
\text { Tower }\end{array}$ & & 0.081 & 0.122 & 0.081 & 0.070 & 0.057 & 0.316 & 0.062 \\
\hline 10 & $\begin{array}{l}\text { Stainless Water } \\
\text { Tower }\end{array}$ & & 0.087 & 0.147 & 0.087 & 0.070 & 0.061 & 0.199 & 0.077 \\
\hline 11 & $\begin{array}{l}\text { Stainless Water } \\
\text { Tower }\end{array}$ & & 0.385 & 0.060 & 0.385 & 0.015 & 0.105 & 0.494 & 0.422 \\
\hline 12 & $\begin{array}{l}\text { Stainless Water } \\
\text { Tower } \\
\end{array}$ & & 0.049 & 0.117 & 0.049 & 0.043 & 0.035 & 0.159 & 0.037 \\
\hline 13 & $\begin{array}{l}\text { Stainless Water } \\
\text { Tower }\end{array}$ & & 0.077 & 0.077 & 0.077 & 0.060 & 0.054 & 0.240 & 0.062 \\
\hline 14 & $\begin{array}{l}\text { Stainless Water } \\
\text { Tower }\end{array}$ & & 0.053 & 0.021 & 0.053 & 0.004 & 0.041 & 0.125 & 0.041 \\
\hline 15 & $\begin{array}{l}\text { Stainless Water } \\
\text { Tower }\end{array}$ & & 0.125 & 0.050 & 0.125 & 0.121 & 0.088 & 0.170 & 0.147 \\
\hline 16 & $\begin{array}{l}\text { Stainless Water } \\
\text { Tower }\end{array}$ & & 1.000 & 1.000 & 1.000 & 0.243 & 0.069 & 1.000 & 1.000 \\
\hline
\end{tabular}




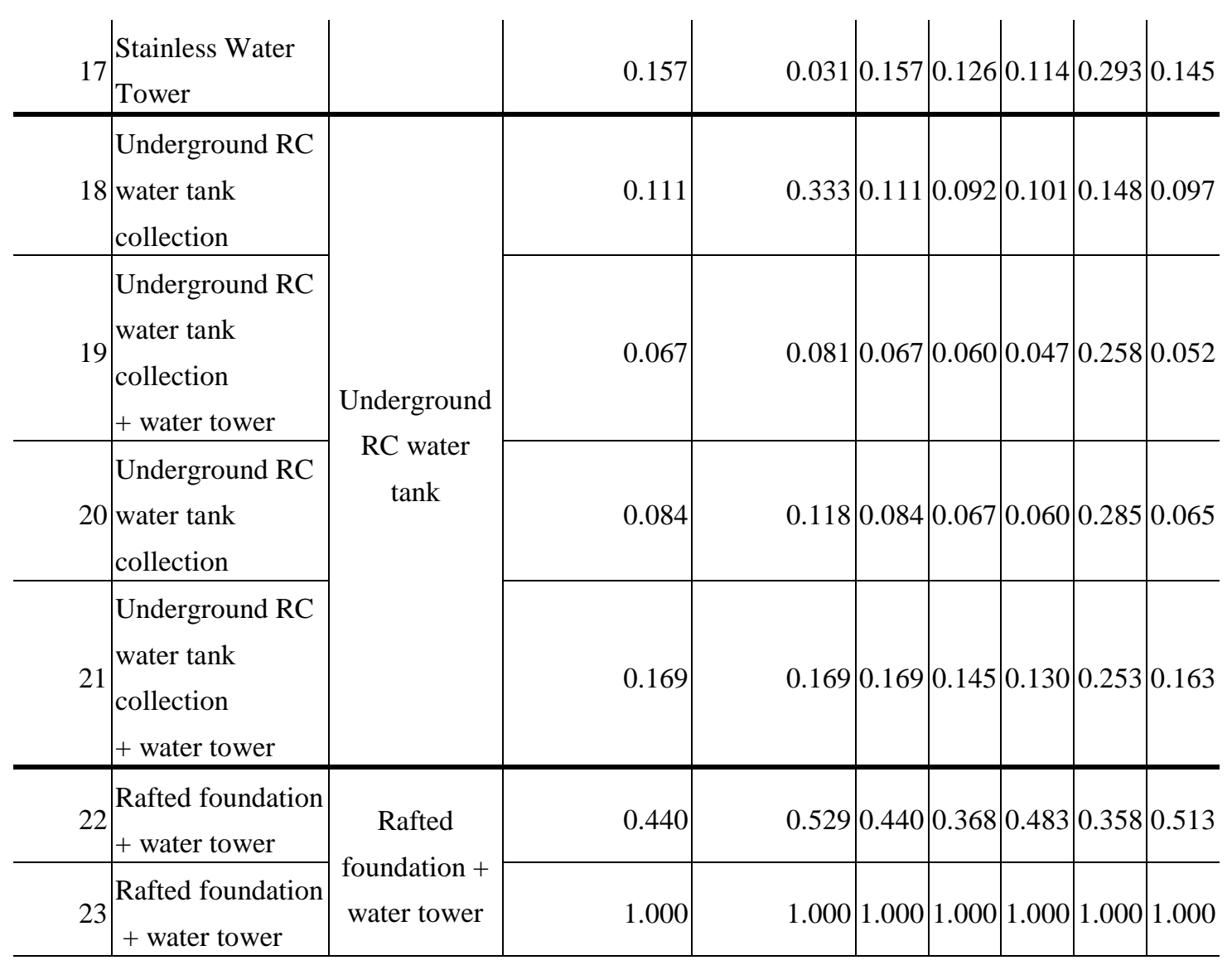

This study categorized projects into sub-groups and has obtained the average rate of efficiency analysis, effectiveness analysis and sensitivity analysis (Table 4 and Fig. 1):

1.- "Efficiency analysis" on average: The rainwater recycle improvement efficiency rates are as follows:

High ground water tank (recycling \& reuse) (0.720), underground RC water tank collection (0.207), high ground water tank with rafted foundation $(0.108)$

2.- "Effectiveness analysis" on average: The rainwater recycle improvement efficiency rates are as follows:

High ground water tank (recycling \& reuse) (0.765), underground RC water tank collection (0.179), high ground water tank with rafted foundation $(0.175)$

3.- "Sensitivity Analysis" (-I1) on average: The rainwater recycle improvement efficiency rates are as follows:

High ground water tank (recycling \& reuse) (0.720), underground RC water tank collection (0.167), high ground water tank with rafted foundation (0.108)

4.- "Sensitivity Analysis" (-I2) on average: The rainwater recycle improvement efficiency rates are as follows:

High ground water tank (recycling \& reuse) (0.684), underground RC water tank collection (0.131), high ground water tank with rafted foundation (0.091)

5.- "Sensitivity Analysis" (-O1) on average: The rainwater recycle improvement efficiency rates are as follows:

High ground water tank (recycling \& reuse) (0.742), underground RC water tank collection (0.085), high ground water tank with rafted foundation $(0.064)$ 
6.- "Sensitivity Analysis" (-O2) on average: The rainwater recycle improvement efficiency rates are as follows:

High ground water tank (recycling \& reuse) (0.679), underground RC water tank collection (0.364), high ground water tank with rafted foundation $(0.236)$

7.- "Sensitivity Analysis" (-O3) on average: The rainwater recycle improvement efficiency rates are as follows:

High ground water tank (recycling \& reuse) (0.757), underground RC water tank collection (0.198), high ground water tank with rafted foundation (0.094)

Table 4. Rainwater (Recycling \& reuse)-Case Group by using SBM Model Analysis score value

\begin{tabular}{|c|c|c|c|c|c|c|c|c|c|}
\hline \multirow[b]{3}{*}{ Code } & \multirow[b]{3}{*}{ Case Group } & \multirow[b]{3}{*}{$\begin{array}{l}\text { (Combine) } \\
\text { DMU Code }\end{array}$} & \multirow[b]{3}{*}{$\begin{array}{c}\text { Efficiency } \\
\text { Average } \\
\text { Rate }\end{array}$} & \multirow[b]{3}{*}{$\begin{array}{c}\text { Effectivenes } \\
\text { s } \\
\text { Average } \\
\text { Rate }\end{array}$} & \multicolumn{5}{|c|}{$\begin{array}{c}\text { Sensitivity Analysis } \\
\text { Average Rate }\end{array}$} \\
\hline & & & & & $-\mathrm{I} 1$ & $-\mathrm{I} 2$ & $-\mathrm{O} 1$ & $-\mathrm{O} 2$ & $-\mathrm{O} 3$ \\
\hline & & & & & 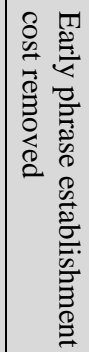 & 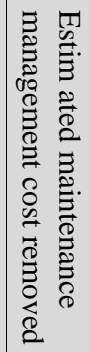 & 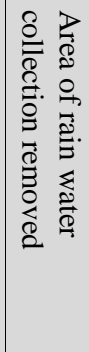 & 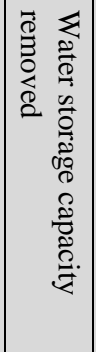 & 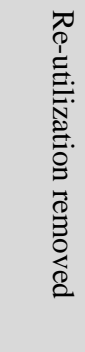 \\
\hline 1. & $\begin{array}{l}\text { High Ground water } \\
\text { tower (Recycling \& } \\
\text { reuse) }\end{array}$ & $1-17$ & 0.207 & 0.179 & 0.167 & 0.131 & 0.064 & 0.364 & 0.198 \\
\hline 2. & $\begin{array}{l}\text { underground RC water } \\
\text { tank collection }\end{array}$ & $18-21$ & 0.108 & 0.175 & 0.108 & 0.091 & 0.085 & 0.236 & 0.094 \\
\hline 3. & $\begin{array}{l}\text { Rafted foundation } \\
\text { High Ground + water } \\
\text { tower }\end{array}$ & $22-23$ & 0.720 & 0.765 & 0.720 & 0.684 & 0.742 & 0.679 & 0.757 \\
\hline
\end{tabular}

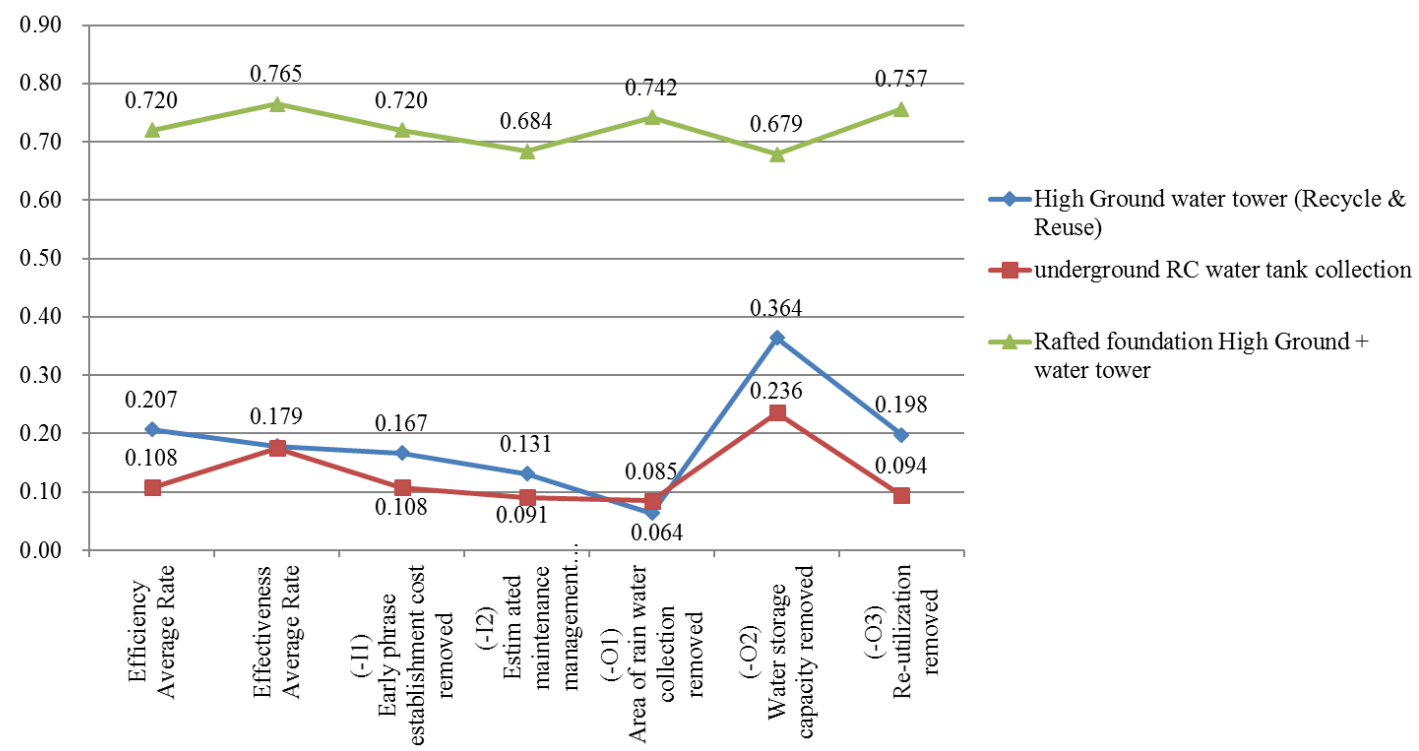

Figure 1. Rainwater (Recycling \& reuse)- Susceptibility analysis 
Overall: Based on the efficiency analysis, the re-utilization of high ground water tank with rafted foundation performs better than High ground water tank (recycling $\&$ reuse) and underground $\mathrm{RC}$ water tank collection. The main advantage is on the storage capacity. There is a minor difference with regard to the re-utilization of High ground water tank (recycling \& reuse) and underground RC water tank collection (Fig. 2).

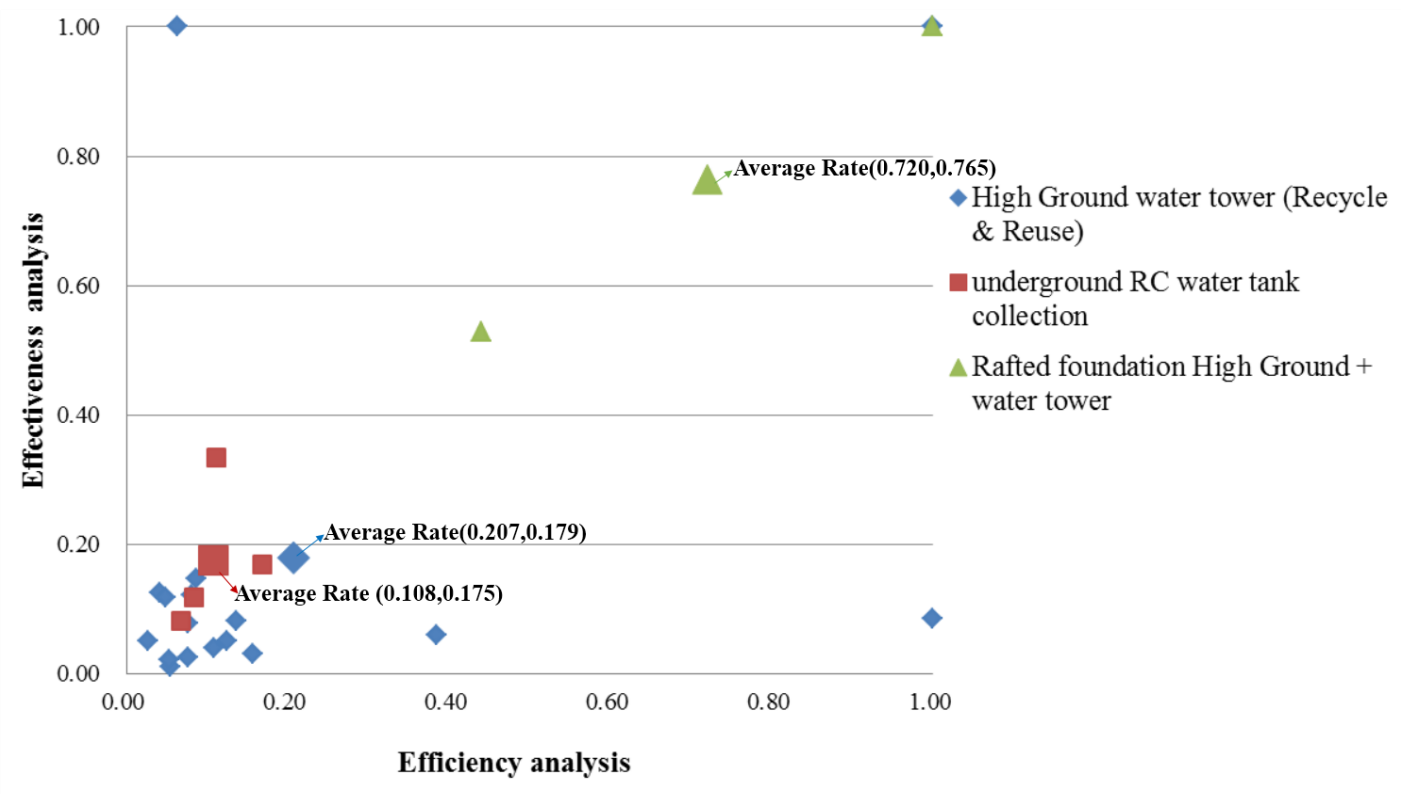

Figure 2. Effectiveness Analysis and Efficiency Analysis scores

\section{Conclusion}

This study conducted a case study of schools which have implemented energy-saving plans, and proposed the improvement checklist which would be suitable for energysaving program for schools in Taiwan. Throughout this study, the "environmental efficiency" concept and the "Data Envelopment Analysis (DEA)" analysis have been implemented. In addition, input/output factors are taken into consideration. Moreover, efficiency analysis, effectiveness analysis, and sensitivity analysis are used to better understand the techniques of localized "energy-saving" method. With the above, analytical models for "water-saving", "healthy-saving" and "energy control" could be established and constructed.

The case study and the analysis have provided an empirical evidence that high ground water tower with rafted foundation shows best efficiency performance (Fig. 2). In practice, the method of high ground water tower with rafted foundation has the advantage of large water storage capacity. However, the disadvantage is the reutilization water source came from underground, and requires extra power to pump the water from bottom to the top. In addition, the gravitational method of supplying water is required. Moreover, in terms of environmental education, the educational observation of rain collection is not easy. Creative observation holes and float balls are required. High ground water tower with rafted foundation is able to collect a large volume of rainwater and therefore the re-utilization of rainwater shall be more diversified. The case study indicated that a flushing toilet takes a large part of water consumption and it shall reuse 
rainwater instead of running water. If allowed by the conditions, watering plants and floor cleaning shall also use rainwater. In addition, approaches such as the close monitoring of water consumption and promoting energy-saving can maximize the effectiveness of rainwater recycling and reuse strategy.

Acknowledgements. Support for this study is gratefully acknowledged from the cases of the Sustainable Campus Renovation Plan by the Ministry of Education, Taiwan.We also thank two anonymous reviewers for their valuable comments and suggestions.

\section{REFERENCES}

[1] Blomberg, J., Henriksson, E., Lundmark, R. (2012): Energy efficiency and policy in Swedish pulp and paper mills: a data envelopment analysis approach. - Energy Policy 42: 569-579.

[2] Chang, D. S., Yang, F. C. (2011): Assessing the power generation, pollution control, and overall efficiencies of municipal solid waste incinerators in Taiwan. - Energy Policy 39(2): 651-663.

[3] Chang, P. L., Hwang, S. N., Cheng, W. Y. (1995): Using data envelopment analysis to measure the achievement and change of regional development in Taiwan. - Journal of Environmental Management 43(1): 49-66.

[4] Liao, Y. T., Chiang, C. M., Liu, K. S., Tzeng, C. T.(2014): Decision-making factors of school building renovations for improving built environment. - Journal of Environmental Protection and Ecology (JEPE) 15.3A: 1246-1254.

[5] Macek, K., Mařík, K. (2012): A methodology for quantitative comparison of control solutions and its application to HVAC (heating, ventilation and air conditioning) systems. Energy 44(1): 117-125.

[6] Magliano, P. N., Murray, F., Baldi, G., Aurand, S., Páez, R. A., Harder, W., Jobbágy, E. G. (2015): Rainwater harvesting in Dry Chaco: Regional distribution and local water balance. - Journal of Arid Environments 123, 93-102.

[7] Song, M., Wang, S., Liu, Q. (2013): Environmental efficiency evaluation considering the maximization of desirable outputs and its application. - Mathematical and Computer Modelling 58(5): 1110-1116.

[8] Tone, K. (2001): A slacks-based measure of efficiency in data envelopment analysis. European journal of operational research: 130(3): 498-509.

[9] UNESCO (1992): Agenda 21: Promoting education, public awareness and training 36:1.

[10] Wang, K., Yu, S., Zhang, W. (2013): China's regional energy and environmental efficiency: A DEA window analysis based dynamic evaluation. - Mathematical and Computer Modelling 58(5): 1117-1127.

[11] Yu, F. W., Chan, K. T. (2012): Improved energy management of chiller systems by multivariate and data envelopment analyses. - Applied energy 92: 168-174. 